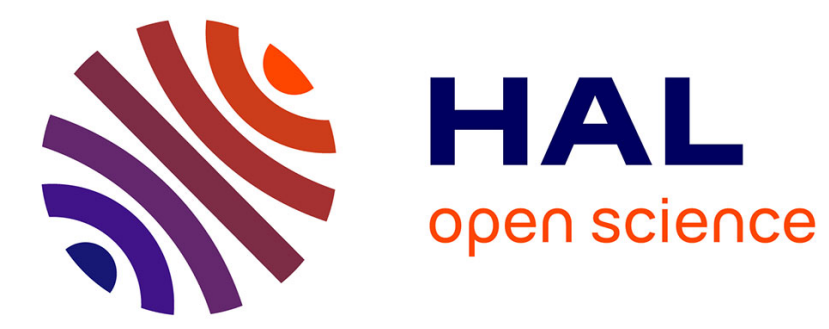

\title{
Thermodynamics of crack nucleation
}

Thomas Carlioz, Luc Dormieux, Éric Lemarchand

\section{To cite this version:}

Thomas Carlioz, Luc Dormieux, Éric Lemarchand. Thermodynamics of crack nucleation. Continuum Mechanics and Thermodynamics, 2020, 10.1007/s00161-020-00863-7 . hal-02537934

\section{HAL Id: hal-02537934 \\ https://hal.science/hal-02537934}

Submitted on 1 Jan 2021

HAL is a multi-disciplinary open access archive for the deposit and dissemination of scientific research documents, whether they are published or not. The documents may come from teaching and research institutions in France or abroad, or from public or private research centers.
L'archive ouverte pluridisciplinaire HAL, est destinée au dépôt et à la diffusion de documents scientifiques de niveau recherche, publiés ou non, émanant des établissements d'enseignement et de recherche français ou étrangers, des laboratoires publics ou privés. 


\title{
Thermodynamics of crack nucleation
}

\author{
Thomas Carlioz • Luc Dormieux · Eric \\ Lemarchand
}

Received: date / Accepted: date

\begin{abstract}
Crack nucleation issue is adressed in a comprehensive micromechanicsbased framework allowing to bridge the $2 \mathrm{D}$ model with the more realistic $3 \mathrm{D}$ representation of a crack. The sudden and abrupt nature of the nucleation process argues in favour of adiabatic conditions rather than isothermal so that the formulation of the energy balance is formulated in terms of internal energy instead of Helmholtz free energy. The proposed theory provides the mean to evaluate the temperature rise as a function of the created entropy at the microscopic scale and the internal energy crack density at the macroscopic one.
\end{abstract}

Keywords crack nucleation, adiabatic conditions, micromechanics, temperature rise, thermodynamics framework

T. Carlioz

6-8 Avenue Blaise Pascal, 77420 Champs-sur-Marne, France

E-mail: thomas.carlioz@enpc.fr

L. Dormieux

6-8 Avenue Blaise Pascal, 77420 Champs-sur-Marne, France

E-mail: luc.dormieux@enpc.fr

E. Lemarchand

6-8 Avenue Blaise Pascal, 77420 Champs-sur-Marne, France

E-mail: eric.lemarchand@enpc.fr 


\section{List of symbols}

$t \quad:$ time

$\boldsymbol{x} \quad: \quad$ macroscopic position vector

$\boldsymbol{z} \quad:$ microscopic position vector

$f \quad$ : volume fraction of microcracks in the REV

$d \quad:$ damage parameter

$\boldsymbol{T} \quad$ : stress vector

$\boldsymbol{\xi} \quad:$ displacement field

$\boldsymbol{\sigma} \quad$ : Cauchy stress tensor

$\varepsilon \quad: \quad$ infinitesimal strain tensor

$\rho \quad:$ mass density

$\ell \quad: \quad$ crack length

$\mathcal{F}(\ell) \quad: \quad 2 \mathrm{D}$ crack subset

$\mathcal{F}^{ \pm}(\ell) \quad: \quad$ upper and lower lips of the crack

$\boldsymbol{N} \quad: \quad$ outer unit normal to the crack upper lip

$\epsilon \quad$ : finite thickness of the macrocrack

$\mathcal{L}_{\epsilon} \quad: \quad 3 \mathrm{D}$ geometrical model of a macrocrack

$\Omega_{0} \quad: \quad$ whole structure, including the crack

$\Omega(\ell) \quad: \quad$ complementary subset of the crack in $\Omega_{0}$

$\partial \Omega_{0} \quad: \quad$ boundaries of $\Omega_{0}$

$\partial \Omega^{T} \quad: \quad$ subset of $\partial \Omega_{0}$ where the stress boundary conditions are defined

$\partial \Omega^{\xi} \quad: \quad$ subset of $\partial \Omega_{0}$ where the displacement boundary conditions are defined

$\rho \boldsymbol{F} \quad:$ mass density force

$\mathcal{C}_{F}(t) \quad: \quad$ scalar time function controlling the time-dependent intensity of the body forces

$\mathcal{C}_{T}(t) \quad: \quad$ scalar time function controlling the time-dependent intensity of the stress boundary conditions

$\mathcal{C}_{\xi}(t) \quad: \quad$ scalar time function controlling the time-dependent intensity of the displacement boundary conditions

$U_{\text {tot }} \quad:$ internal energy of $\Omega_{0}$

$U_{\text {bulk }} \quad: \quad$ internal energy of $\Omega(\ell)$

$U_{\mathcal{F}} \quad: \quad$ internal energy of $\mathcal{F}$

$\Psi \quad$ : Helmholtz free energy

$K \quad$ : kinetic energy

$\mathcal{P}_{e} \quad: \quad$ rate of work done by external forces acting on the system in its actual motion

$\Phi(\boldsymbol{\xi}) \quad$ : $\quad$ work of the given external forces

$G_{c} \quad: \quad$ critical energy release rate

$u \quad:$ internal energy density

$\psi \quad$ : Helmholtz free energy density

$s \quad$ : entropy density

$\stackrel{\circ}{s}_{c r} d t \quad: \quad$ volume entropy created between time $t$ and $t+d t$

$\stackrel{\circ}{q} \quad$ : heat input density

$r \quad: \quad$ heat supply density

$\boldsymbol{q} \quad:$ heat flow vector

D : dissipation per unit volume

$T \quad$ : temperature

$T^{0} \quad$ : temperature of reference

$T^{0} c \quad$ : heat capacity density

$T^{0} \boldsymbol{k} \quad$ : $\quad$ strain latent heat density

$\mathbb{C}_{\text {iso }} \quad$ : isothermal elastic tensor

$\mathbb{C}_{a d} \quad: \quad$ adiabatic elastic tensor

$c^{\text {th }} \quad:$ thermal diffusivity

$t^{c} \quad:$ characteristic time of heat transfer

$U \quad$ : macroscopic internal energy density

$U^{F D} \quad$ : internal energy density of a fully damaged material

$\tau \quad:$ temperature variation with respect to the reference state

$\boldsymbol{E} \quad$ : average strain of the REV

$\mathbb{C}^{\text {hom }}(d)$ : macroscopic homogenized elasticity of a microcracked domain

$\boldsymbol{\Sigma} \quad$ : macroscopic stress

S : macroscopic entropy density

II : symetrical fourth order identity tensor

$\mathbb{A} \quad: \quad$ strain concentration tensor

$g_{c} \quad:$ material constant characterizing the dissipative process

$G_{c}^{a d} \quad: \quad$ critical energy release rate in adiabatic context 


\section{Introduction}

The usual energy balance proposed for crack propagation (e.g. [6], [14], [5], [3]) is formulated under isothermal conditions. This assumption can be justified if the propagation is sufficiently slow (see [16]) while the structure is in contact with an isothermal reservoir. However, crack nucleation is a sudden and abrupt phenomenon which is expected to involve a temperature rise along the nucleated crack. In this context, isothermal assumption may be a rough approximation. This problem seems not to have focused the attention since the temperature rise itself and its determination are not considered (see [12], [13] and [8]). The starting point of this study is to consider that the adiabatic framework is better suited to capture the nucleation process rather than the isothermal one.

In isothermal conditions, the fundamental Clausius-Duhem inequality introduces the concept of dissipation defined as the difference between the mechanical work and the Helmholtz free energy variation. In fact, the concept of dissipation is intrinsically related to isothermal conditions and is not well suited for adiabatic conditions. From a thermodynamical point of view, the irreversible phenomenon is responsible for a heat creation while there is no heat transfer in adiabatic conditions. Hence, there is a heat accumulation along the crack nucleation. If we have in mind to resort to a description of the crack as a surface, the heat accumulation suggests that an internal energy surface density should be, in adiabatic conditions, the counterpart of dissipation in isothermal conditions.

It should be recalled that the classical $2 \mathrm{D}$ geometrical model for cracks is primarily a mathematical model which is introduced in order to derive analytical solutions such as stress intensity factors, etc. It seems however difficult to understand the thermodynamics at stake with such a description. More precisely, if we want to clarify the physics of this internal energy surface density, a 3D description, in which the crack is given a finite width, is due. The proposed point of view is to represent the nucleated crack as a region in which the material has reached the final stage of a damage process. To some extent, this idea is also shared by the damage gradient models (see [11]). In other words, we look for a micromechanicsbased thermodynamics theory of damage in order to bridge the mathematical $2 \mathrm{D}$ model with the 3D real nature of the crack.

Sections 2 and 3 are dedicated to the presentation of the thermodynamics principles, specifically in adiabatic conditions. The internal energy is identified as the relevant quantity for deriving the energy balance of the crack nucleation process. Section 4 is devoted to the micromechanics-based interpretation of the macroscopic adiabatic crack. The macroscopic internal energy is derived, allowing for the derivation of both the state equations of the damaged REV and the macroscopic thermodynamics driving force related to the damage variable (section 5 ). In section 6 , a $2 \mathrm{D}$ representation of the crack is proved to be consistent with the $3 \mathrm{D}$ model. The question of the dissipation mechanism is also adressed and the temperature rise associated with the adiabatic crack nucleation is proposed. 


\section{Macroscopic scale description}

\subsection{Crack description}

To begin with, we will apply the thermodynamics principles at the macroscopic scale, meaning to the scale of the considered structure. For the sake of simplicity, we assume that the crack length can be defined by a unique scalar parameter $\ell$. At a given time $t$, the structure $\Omega_{0}$ is assumed to be partitioned in two subsets :

$$
\Omega_{0}=\Omega(\ell) \cup \mathcal{F}(\ell)
$$

where $\mathcal{F}(\ell)$ is the crack and $\Omega(\ell)$ is the complementary subset of the crack in $\Omega_{0}$. In this section, the crack is modelled as a surface, that is a $2 \mathrm{D}$ object. It means that the thickness of the crack is here not explicitly considered. However, it is useful to keep in mind that the crack has indeed a finite thickness and is made of elementary pieces without mechanical link between them. Thus, the energy of the crack is the energy of this $3 \mathrm{D}$ object. Since the crack has no stiffness, this energy does not include elastic contribution. This means that the energy stored in the crack is thermal in nature.

\subsection{Macroscopic scale thermodynamics}

The following approach is very similar to the one classically applied in isothermal conditions in order to identify the thermodynamics driving force associated with a crack propagation (see for instance [3]). The reasoning will be held on the whole structure $\Omega_{0}$. Its boundary $\partial \Omega_{0}$ does not depend neither on $\ell$ nor on time. It can be split into two complementary parts, namely $\partial \Omega^{T}$ (resp. $\partial \Omega^{\xi}$ ) where stress (resp. displacement) boundary conditions are defined, such that $\partial \Omega_{0}=\partial \Omega^{T} \cup \partial \Omega^{\xi}$ (see Figure 1). It is emphasized that the boundary of $\Omega(\ell)$ can be split into $\partial \Omega_{0}$ and $\mathcal{F}(\ell)=\mathcal{F}^{+}(\ell) \cup \mathcal{F}^{-}(\ell)$ where $\mathcal{F}^{+}(\ell)$ (resp. $\mathcal{F}^{-}(\ell)$ ) is the upper (resp. lower) crack lip. The unit vector normal to the upper (resp. lower) crack lip $\mathcal{F}^{+}(\ell)$ (resp. $\mathcal{F}^{-}(\ell)$ ) is denoted by $\boldsymbol{N}$ (resp. $-\boldsymbol{N}$ ). The crack lips are not necessarily considered as stress free in order to take into account closed shear cracks. Besides, the crack lips evolve during the propagation process.

For the sake of simplicity, a radial loading is considered. It involves scalar time functions $\mathcal{C}_{F}(t), \mathcal{C}_{T}(t)$ and $\mathcal{C}_{\xi}(t)$ controlling the time-dependent intensity of the body forces, the stress and the displacement boundary conditions respectively. The mass density of force applied to the system can be written as $\rho \boldsymbol{F}(\boldsymbol{x}, t)=\rho \mathcal{C}_{F}(t) \boldsymbol{F}^{0}(\boldsymbol{x})$ where $\boldsymbol{x}$ is the position vector and $\rho$ is the mass density. The boundary conditions on the stress vector $\boldsymbol{T}$ and on the displacement $\boldsymbol{\xi}$ also read :

$$
\forall \boldsymbol{x} \in \partial \Omega^{T}: \boldsymbol{T}(\boldsymbol{x}, t)=\mathcal{C}_{T}(t) \boldsymbol{T}^{0}(\boldsymbol{x}) \quad, \quad \forall \boldsymbol{x} \in \partial \Omega^{\xi}: \boldsymbol{\xi}(\boldsymbol{x}, t)=\mathcal{C}_{\xi}(t) \boldsymbol{\xi}^{0}(\boldsymbol{x})
$$

Let $K$ be the kinetic energy of the considered system. The first thermodynamics principle applied to $\Omega_{0}$ in adiabatic conditions is:

$$
\dot{U}_{t o t}+\dot{K}=\mathcal{P}_{e}
$$




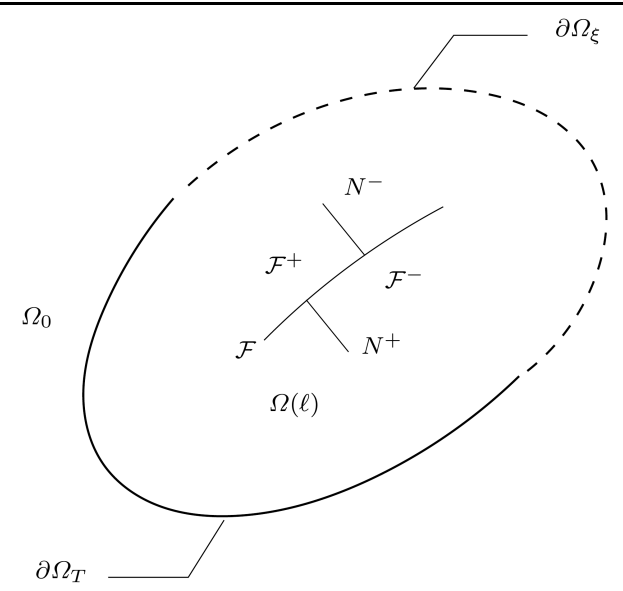

Fig. 1 Schematic representation of the structure $\Omega_{0}$ including the crack $\mathcal{F}(\ell)$

where $\mathcal{P}_{e}$ is the rate of work done by external forces acting on the system in its actual motion:

$$
\mathcal{P}_{e}=\int_{\Omega_{0}} \rho \boldsymbol{F} \cdot \dot{\boldsymbol{\xi}} d V+\int_{\partial \Omega_{0}} \boldsymbol{T} \cdot \dot{\boldsymbol{\xi}} d A
$$

Recalling the considered radial loading path, it also reads ${ }^{1}$ :

$$
\mathcal{P}_{e}=\mathcal{C}_{F}(t) \int_{\Omega(\ell)} \rho \boldsymbol{F}^{0} \cdot \dot{\boldsymbol{\xi}} d V+\mathcal{C}_{T}(t) \int_{\partial \Omega^{T}} \boldsymbol{T}^{0} \cdot \dot{\boldsymbol{\xi}} d A+\dot{\mathcal{C}}_{\xi}(t) \int_{\partial \Omega^{\xi}} \boldsymbol{T} \cdot \boldsymbol{\xi}^{0} d A
$$

In the first principle (3), $U_{\text {tot }}$ corresponds to the total internal energy stored in $\Omega(\ell) \cup \mathcal{F}(\ell)$ at time $t$. This quantity being extensive we derive :

$$
U_{\text {tot }}=U_{\text {bulk }}+U_{\mathcal{F}}
$$

where $U_{\text {bulk }}$ (resp. $U_{\mathcal{F}}$ ) is the internal energy stored into $\Omega(\ell)($ resp. $\mathcal{F}(\ell)$ ).

At a given time $t$, the displacement field solution of the problem involving a crack of length $\ell(t)$ and a loading $\mathcal{C}(t)$ will be referred to as $\boldsymbol{\xi}(t)=\boldsymbol{\xi}(\mathcal{C}(t), \ell(t))$. Alongside the loading $\mathcal{C}(t)$, the crack length $\ell(t)$ appears as a second time-dependent parameter. Thus, it is convenient to introduce the partial time derivative of a physical quantity $a$ at a fixed crack geometry :

$$
\dot{a}_{\mid \ell}=\frac{\partial a}{\partial \mathcal{C}} \dot{\mathcal{C}}_{\mid \ell}
$$

Hence, the displacement rate $\dot{\boldsymbol{\xi}}$ in the integrals of (5) can be computed as the sum of two terms :

$$
\dot{\boldsymbol{\xi}}=\dot{\boldsymbol{\xi}}_{\mid \ell}+\frac{\partial \boldsymbol{\xi}}{\partial \ell} \mid \mathcal{C}
$$

Using (8) in (5) allows to split $\mathcal{P}_{e}$ into two terms :

$$
\mathcal{P}_{e}=\mathcal{P}_{e \mid \ell}+\mathcal{P}_{e \mid \mathcal{C}}
$$

\footnotetext{
1 The volume $\Omega(\ell)$ over which is taken the integral in (5) can be replaced by $\Omega_{0}$ if needed
} 
where $\mathcal{P}_{e \mid \ell}$ stands for the rate of work done by external forces at fixed geometry:

$$
\begin{aligned}
\mathcal{P}_{e \mid \ell}=\left(\mathcal{C}_{F}(t) \int_{\Omega(\ell)} \rho \boldsymbol{F}^{0} . \dot{\boldsymbol{\xi}}_{\mid \ell} d V+\mathcal{C}_{T}(t) \int_{\partial \Omega^{T}} \boldsymbol{T}^{0} . \dot{\boldsymbol{\xi}}_{\mid \ell} d A\right) & \\
& +\dot{\mathcal{C}}_{\xi}(t)\left(\int_{\partial \Omega^{\xi}} \boldsymbol{T} . \boldsymbol{\xi}^{0} d A\right)
\end{aligned}
$$

The crack length and thus the geometry being fixed, it is possible to use the principle of virtual rate of work. It can be computed as :

$$
\mathcal{P}_{e \mid \ell}-\dot{K}_{\mid \ell}=\int_{\Omega(\ell)} \sigma: \dot{\varepsilon}_{\mid \ell} d V
$$

where $\varepsilon$ is the infinitesimal strain tensor. Based on local thermodynamics, the state equations for a linear reversible elastic medium (given in (25)) yield:

$$
\boldsymbol{\sigma}: \dot{\varepsilon}_{\mid \ell}=\dot{u}_{\mid \ell}-\frac{\partial u}{\partial s} \dot{s}_{\mid \ell}
$$

where $u$ stands for the internal energy density. The considered evolution being reversible when $\ell$ is fixed and the conditions being locally adiabatic, the evolution is in fact isentropic, that is $\dot{s}_{\mid \ell}=0$. Consequently, the integral form of (12) together with (11) yields:

$$
\mathcal{P}_{e \mid \ell}-\dot{K}_{\mid \ell}=\dot{U}_{t o t \mid \ell}
$$

Then, introducing (13) in (3):

$$
\mathcal{P}_{e \mid \mathcal{C}}-\frac{\partial K}{\partial \ell}{ }_{\mid \mathcal{C}} \dot{\ell}=\frac{\partial U_{t o t}}{\partial \ell}{ }_{\mid \mathcal{C}} \dot{\ell}
$$

$\mathcal{P}_{e \mid \mathcal{C}}$ may be interpreted as the rate of work of the external forces applied on the system during the crack propagation at a given and constant loading :

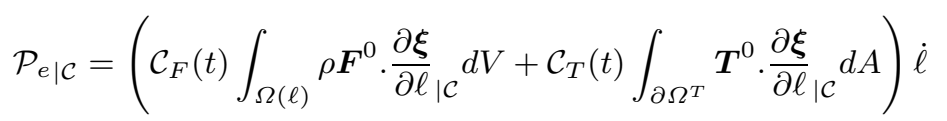

Introducing $\Phi(\boldsymbol{\xi})$ as the work of the given external forces:

$$
\Phi(\boldsymbol{\xi})=\mathcal{C}_{F}(t) \int_{\Omega(\ell)} \rho \boldsymbol{F}^{0} \cdot \boldsymbol{\xi} d V+\mathcal{C}_{T}(t) \int_{\partial \Omega^{T}} \boldsymbol{T}^{0} \cdot \boldsymbol{\xi} d A
$$

it is readily seen that :

$$
\mathcal{P}_{e \mid \mathcal{C}}=\frac{\partial \Phi}{\partial \ell}{ }_{\mathcal{C}} \dot{\ell}
$$

Combining (14) and (17) yields :

$$
\frac{\partial}{\partial \ell}\left(U_{t o t}+K-\Phi\right)_{\mid \mathcal{C}}=0
$$

Equation (18) stands for the conservation of the mechanical energy in a propagation or nucleation process occuring at fixed loading $\mathcal{C}$. This property is a bit disturbing since its counterpart in isothermal conditions is :

$$
-\frac{\partial}{\partial \ell}(\Psi+K-\Phi)_{\mid \mathcal{C}}=G_{c}
$$


where $\Psi$ stands for the Helmholtz free energy of the structure, $G_{c}$ is the critical energy release rate and $G_{c} \dot{\ell}$ the dissipation in isothermal conditions. In equation (18), the dissipation does not appear directly. However, we have to keep in mind that this dissipation has been introduced precisely in isothermal conditions. We will show that even if this notion is relevant in isothermal conditions, it is more convenient and more natural, in adiabatic evolutions, to deal with the classical notion of created entropy, which is in fact hidden in the definition of the dissipation. Following (6), $U_{\text {tot }}$ stands for the total internal energy of the structure. Owing to (1), it is readily seen that $U_{\text {tot }}^{0}=U_{\text {bulk }}^{0}+U_{\mathcal{F}}^{0}=c^{\text {ste }}$. Consequently, (18) can be rewritten as :

$$
-\frac{\partial}{\partial \ell}\left(U_{b u l k}-U_{b u l k}^{0}+K-\Phi\right)_{\mid \mathcal{C}}=\frac{\partial}{\partial \ell}\left(U_{\mathcal{F}}-U_{\mathcal{F}}^{0}\right)_{\mid \mathcal{C}}
$$

Exponent 0 stands for the quantity taken at the initial time (or any time of reference) and $U_{\text {bulk }}^{0}$ (resp. $U_{\mathcal{F}}^{0}$ ) stands for the internal energy contained at this initial time in the subset that now corresponds to $\Omega(\ell)$ (resp. $\mathcal{F}(\ell)$ ). We will later show that the right hand side of this last equation accounts for the notion of created entropy and will be regarded as a material constant. As explained previously, the crack has a heat capacity. Therefore, the way the dissipated energy is handled constitutes the very difference between isothermal an adiabatic conditions:

- in isothermal conditions, the dissipated energy propagates in the medium through thermal diffusion process,

- in adiabatic conditions, the dissipated energy is stored in the crack domain since it cannot propagate at the time scale at stake.

Prior and after nucleation, the Helmholtz free energy of the crack domain is negligible $\left(\Delta \Psi_{\mathcal{F}}=0\right)$. As such, there is no reason to make a distinction between $\Psi_{\text {tot }}$ and $\Psi_{b u l k}$. In contrast, in adiabatic conditions, the internal energy increase of the crack domain corresponds to the energy dissipated along the nucleation process.

\section{Local macroscopic scale thermodynamics}

The present section is devoted to the formulation of the left hand side of (20) in terms of local energy densities.

\subsection{Thermal exchange and dissipation}

Restricting the study to the case where the local heat exchange can be described by a heat supply density $r$ and a heat flow vector $\boldsymbol{q}$ (e.g. [15]), the heat input density $\stackrel{\circ}{q}$ is defined as:

$$
\stackrel{\circ}{q}=r-\operatorname{div} \boldsymbol{q}
$$

In addition, the variation of the entropy density between time $t$ and $t+d t$ reads:

$$
\frac{d s}{d t}=\dot{s}=\frac{r}{T}-\operatorname{div}\left(\frac{\boldsymbol{q}}{T}\right)+\frac{\delta s_{c r}}{d t}=\frac{\stackrel{\circ}{q}}{T}+\frac{1}{T^{2}} \boldsymbol{q} \cdot \operatorname{grad} T+\stackrel{\circ}{s}_{c r}
$$


where $\stackrel{\circ}{s}_{c r} d t(\geq 0)$ stands for the volume entropy created between time $t$ and $t+d t$. The hypothesis made over the characteristic time implies that the behaviour is adiabatic over every macroscopic subsystem. This implies that $r=0$ and $\boldsymbol{q}=\mathbf{0}$. The Clausius-Duhem inequality can then be written in adiabatic conditions as :

$$
D=\boldsymbol{\sigma}: \dot{\varepsilon}-\dot{u}+T \dot{s} \geq 0
$$

where $D=T \stackrel{\circ}{s}_{c r}$ stands for the dissipation per unit volume.

\subsection{Internal energy density}

Since the evolution is adiabatic and the behaviour outside the created crack is linear thermoelastic, the internal energy density $u$ solely depends on the state variables $\varepsilon$ and $s$ :

$$
u(\varepsilon, s)=u^{0}+\boldsymbol{\sigma}^{0}: \varepsilon+\frac{1}{2} \varepsilon: \mathbb{C}_{a d}: \varepsilon+T^{0}\left(s-s^{0}\right)+\frac{\left(s-s^{0}\right)^{2}}{2 c}-\frac{\left(s-s^{0}\right)}{c} \boldsymbol{k}: \boldsymbol{\varepsilon}
$$

The behaviour being reversible, the dissipation $D$ in (23) is equal to zero. We can then derive the two thermoelastic state equations:

$$
\left\{\begin{array}{l}
\boldsymbol{\sigma}=\frac{\partial u}{\partial \varepsilon}=\boldsymbol{\sigma}^{0}+\mathbb{C}_{a d}: \varepsilon-\frac{\left(s-s^{0}\right)}{c} \boldsymbol{k} \\
T=\frac{\partial u}{\partial s}=T^{0}+\frac{1}{c}\left(\left(s-s^{0}\right)-\boldsymbol{k}: \boldsymbol{\varepsilon}\right)
\end{array}\right.
$$

Those state equations allow to identify the physical meaning of the (thermoelastic) material constants introduced in (24). If $T^{0}$ is the temperature of reference then $T^{0} c$ can be interpreted as a heat capacity density, $T^{0} \boldsymbol{k}$ stands for the strain latent heat density of the uncracked material and $\mathbb{C}_{a d}$ is the adiabatic elastic tensor of the uncracked material. The latter is linked to the usual isothermal elastic tensor by :

$$
\mathbb{C}_{a d}=\mathbb{C}_{i s o}+\frac{1}{c} \boldsymbol{k} \otimes \boldsymbol{k}
$$

For a volume element that does not belong to the created crack region, the evolution is both adiabatic and reversible. As such it is in fact isentropic. Consequently, its internal energy density can be computed as :

$$
u=u^{0}+\sigma^{0}: \varepsilon+\frac{1}{2} \varepsilon: \mathbb{C}_{a d}: \varepsilon
$$

With this last result, equation (20) takes the form :

$$
-\frac{\partial}{\partial \ell}\left(\int_{\text {bulk }}\left(\sigma^{0}: \varepsilon+\frac{1}{2} \varepsilon: \mathbb{C}_{a d}: \varepsilon\right) d V+K-\Phi\right)_{\mid \mathcal{C}}=\frac{\partial}{\partial \ell}\left(U_{\mathcal{F}}-U_{\mathcal{F}}^{0}\right)_{\mid \mathcal{C}}
$$

Letting aside the kinetic term $K$, the left hand side of this expression is similar to a potential energy in which the elastic term is calculated with the adiabatic elastic tensor instead of the isothermal one. As such it is referred to as a potential energy. Except for the fact that the elastic tensor refers to adiabatic conditions, (28) is identical to the isothermal one. 
4 Micromechanical approach for a macroscopic adiabatic crack

The purpose of this section is to show how the right hand side of (28) can be linked to a notion of dissipation or more precisely to a notion of irreversibility.

4.1 The micromechanical process leading to a macroscopic crack

Physically speaking, a macroscopic crack in its final stage (full damage process, see Figure 2, right side) is at some scale a set of elementary pieces without mechanical link between them. Therefore, in order to provide a thermodynamics approach of the process yielding the macrocrack, a 3D geometrical model is due, where the macrocrack appears as a layer $\mathcal{L}_{\epsilon}$ with its finite thickness $\epsilon$. Let us introduce the density of internal energy $U^{F D}$ corresponding to full damage :

$$
U_{\mathcal{F}}=\int_{\mathcal{L}_{\epsilon}} U^{F D} d V
$$

In order to sort out the structure of the internal energy of the macrocrack and hence have access to $U^{F D}$ (and further to $U_{\mathcal{F}}$ ), we have to detail a micromechanical mechanism starting from the sound material and ending when the elastic stiffness vanishes. Prior to this final stage, the material domain which is going to become the nucleated macrocrack is the $3 \mathrm{D}$ structure $\mathcal{L}_{\epsilon}$ subjected to damage process. The damage process inside $\mathcal{L}_{\epsilon}$ is described by a time-increasing density of microcracks (see Figure 2, left side). As opposed to the macroscopic crack, the microcracks have neither entropy nor heat capacity. This amounts to saying that microcracks can be viewed as flat pores. The constitutive material inside $\mathcal{L}_{\epsilon}$ is linear elastic perfectly brittle.
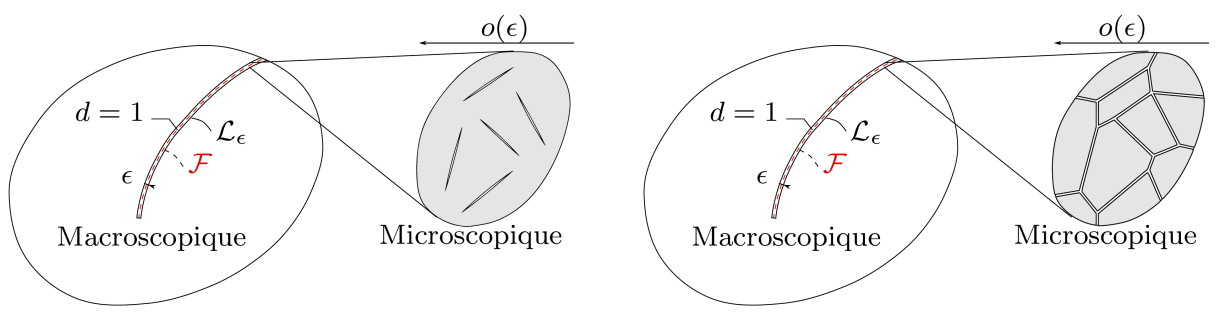

Fig. 2 On the left : The transitory state toward full damage. On the right : 3D representation of a macroscopic crack as a layer of thickness $\epsilon$ of a fully damaged material.

Let $t_{\text {macro }}^{c}$ (resp. $t_{\text {micro }}^{c}$ ) be the characteristic time of the heat transfer operating at the macroscopic (resp. microscopic) scale. An order of magnitude of such quantities takes the form $\frac{l^{2}}{c^{t h}}$ where $l$ is the relevant characteristic length at the scale considered and $c^{t h}$ is the thermal diffusivity. The nucleation characteristic time $t_{n u c l}^{c}$ is supposed to be small as compared to $t_{\text {macro }}^{c}$, but still large as compared to $t_{\text {micro }}^{c}$. This amounts to saying :

$$
\frac{l_{\text {micro }}{ }^{2}}{c_{\text {micro }}^{t h}}=t_{\text {micro }}^{c} \ll t_{\text {nucl }}^{c} \ll t_{\text {macro }}^{c}=\frac{l_{\text {macro }}^{2}}{c_{\text {macro }}^{t h}}
$$


where the microscopic and macroscopic thermal diffusivities $c_{\text {micro }}^{\text {th }}$ and $c_{\text {macro }}^{\text {th }}$ are believed to be of the same order of magnitude. This framework justifies the definition of a uniform temperature at the scale of the REV.

\subsection{Homogenization problem and macroscopic quantities}

In order to derive the elastic moduli of the domain getting damaged, which is going to be the nucleated crack, an REV of the microcracked domain has to be considered inside $\mathcal{L}_{\epsilon}$ (see Figure 3). $\boldsymbol{E}$ stands for the average strain over the REV

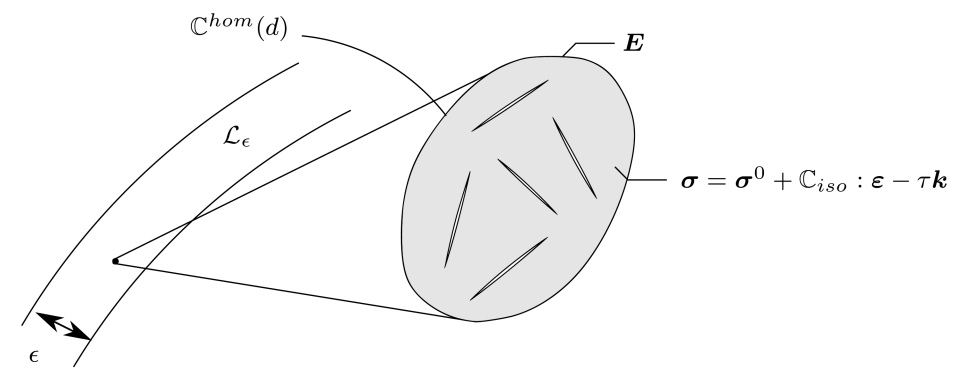

Fig. 3 Representation of the micromechanics-based damage model of the macrocrack

and $f$ is the volume fraction of the microcracks in the REV. For forthcoming purposes, let us recall that classical homogenization theories (e.g. [3], [10]) prove that the relevant damage parameter involved in the definition of the homogenized elastic moduli is not $f$ but rather the crack density parameter $\epsilon^{\star}$ [1]. Assuming a uniform distribution of orientation of identical oblate spheroidal microcracks with diameter $2 a$ and aperture $2 c$, the latter is defined as $\epsilon^{\star}=\mathcal{N} a^{3}$ where $\mathcal{N}$ is the number of microcracks per unit volume of REV. Introducing the (uniform) microcrack aspect ratio $X=c / a$, the microcracks volume fraction is related to the crack density parameter according to $f=\frac{4}{3} \pi X \epsilon^{\star}$. As $X \rightarrow 0$ for cracks, the latter provides a justification of the fact that $f$ is usually considered as an infinitesimal parameter satisfying $f \rightarrow 0$. The damage parameter $d \in[0,1]$ is thus defined as:

$$
d=\frac{\epsilon^{\star}}{\epsilon_{c r}^{\star}}
$$

where $\epsilon_{c r}^{\star}$ corresponds to the value of the crack density parameter for which the full damage state is reached. Its value is determined according to the micromechanics model used for describing the damage process.

The solid matrix will be made of a thermoelastic material whose state equations are given by (25) and whose microscopic internal energy density is computed as (24). Since the temperature is a controlled state variable it is more convenient to 
consider the state equations:

$$
\left\{\begin{array}{l}
\boldsymbol{\sigma}=\frac{\partial \psi}{\partial \varepsilon}=\boldsymbol{\sigma}^{0}+\mathbb{C}_{i s o}: \boldsymbol{\varepsilon}-\boldsymbol{k} \tau \\
s=-\frac{\partial \psi}{\partial T}=s^{0}+c \tau+\boldsymbol{k}: \boldsymbol{\varepsilon}
\end{array}\right.
$$

where $\tau$ is the temperature variation with respect to the reference state, $\mathbb{C}_{\text {iso }}$ is the isothermal elastic tensor which is related to the adiabatic one $\mathbb{C}_{a d}$ according to $(26) . \psi$ is the microscopic Helmholtz free energy density:

$$
\psi=\psi^{0}+\boldsymbol{\sigma}^{0}: \varepsilon+\frac{1}{2} \varepsilon: \mathbb{C}_{i s o}: \varepsilon-\tau \boldsymbol{k}: \boldsymbol{\varepsilon}-s^{0} \tau-\frac{c}{2} \tau^{2}
$$

It is linked to the internal energy density $u$ according to a Legendre transform $u=\psi+T s$. The thermoelastic material parameters and the reference state of stress $\sigma^{0}$ are assumed uniform at the REV scale. Let $\boldsymbol{z}$ be the microscopic position vector and $\chi_{s}(\boldsymbol{z})$ the solid matrix indicator function. Let also $\boldsymbol{\sigma}^{p}(\boldsymbol{z})$ be the second order tensor field defined as :

$$
\boldsymbol{\sigma}^{p}(\boldsymbol{z})=\left(\boldsymbol{\sigma}^{0}-\boldsymbol{k} \tau\right) \chi_{s}(\boldsymbol{z})
$$

and $\mathbb{C}(\boldsymbol{z})$ the fourth order tensor given by :

$$
\mathbb{C}(\boldsymbol{z})=\mathbb{C}_{i s o} \chi_{s}(\boldsymbol{z})
$$

Taking advantage of (34) and (35) the microscopic solid matrix first state equation can then be expanded to the whole REV as :

$$
\boldsymbol{\sigma}=\mathbb{C}(\boldsymbol{z}): \boldsymbol{\varepsilon}+\boldsymbol{\sigma}^{p}(\boldsymbol{z})
$$

It is convenient at this stage to define several macroscopic quantities. The macroscopic stress will be referred to as $\boldsymbol{\Sigma}$. By definition, it is equal to the average stress over the REV. The macroscopic entropy density will be denoted by $S$. The entropy being extensive, $S$ is defined as the average over the REV of the microscopic solid matrix entropy density $s$. We thus have:

$$
\boldsymbol{\Sigma}=\overline{\boldsymbol{\sigma}} \quad, \quad S=(1-f) \bar{s}^{s}
$$

where $\bar{a}$ (resp. $\bar{a}^{\alpha}$ ) stands for the volume average of $a$ over the REV (resp. the phase $\alpha)$.

\subsection{First macroscopic state equation}

According to Levin's theorem [3], the macroscopic stress reads:

$$
\boldsymbol{\Sigma}=\overline{\mathbb{C}: \mathbb{A}}: \boldsymbol{E}+\overline{\boldsymbol{\sigma}^{p}: \mathbb{A}}
$$

where the local strain $\mathbb{A}(\boldsymbol{z}): \boldsymbol{E}$ is the solution of the linear subproblem defined by uniform strain boundary conditions $(\xi=\boldsymbol{E} \cdot \boldsymbol{z}$ ) (see. Figure 3) and no prestress $\left(\boldsymbol{\sigma}^{p}=0\right)$. Due to the strain average rule, the concentration tensor $\mathbb{A}(\boldsymbol{z})$ complies with the consistency rule:

$$
\mathbb{I}=\overline{\mathbb{A}}=f \overline{\mathbb{A}}^{f}+(1-f) \overline{\mathbb{A}}^{s}
$$


where $\mathbb{I}$ is the symetrical fourth order identity tensor. Recalling (34) and (35), (38) gives:

$$
\boldsymbol{\Sigma}=(1-f) \mathbb{C}_{\text {iso }}: \overline{\mathbb{A}}^{s}: \boldsymbol{E}+(1-f)\left(\boldsymbol{\sigma}^{0}-\boldsymbol{k} \tau\right): \overline{\mathbb{A}}^{s}
$$

The homogenized isothermal elastic tensor $\mathbb{C}^{h o m}=(1-f) \mathbb{C}_{i s o}: \overline{\mathbb{A}}^{s}$ is thus derived. Introducing the elastic compliance tensor $\mathbb{S}_{i s o}=\left(\mathbb{C}_{i s o}\right)^{-1}$, we also have:

$$
(1-f) \overline{\mathbb{A}}^{s}=\mathbb{S}_{\text {iso }}: \mathbb{C}^{h o m} \quad, \quad f \overline{\mathbb{A}}^{f}=\mathbb{I}-\mathbb{S}_{\text {iso }}: \mathbb{C}^{h o m}
$$

where (39) has been used. In addition, the following quantities are introduced

$$
\boldsymbol{\Sigma}^{\text {res }}=\boldsymbol{\sigma}^{0}: \mathbb{S}_{i s o}: \mathbb{C}^{h o m} \quad, \quad \boldsymbol{K}=\boldsymbol{k}: \mathbb{S}_{\text {iso }}: \mathbb{C}^{\text {hom }}
$$

so that the macroscopic state equation reads:

$$
\boldsymbol{\Sigma}=\boldsymbol{\Sigma}^{r e s}+\mathbb{C}^{h o m}: \boldsymbol{E}-\boldsymbol{K} \tau
$$

The involved macroscopic parameters depend on the damage level through $\mathbb{C}^{\text {hom }}(d)$. As such, they are evolving from the sound state to the end of the damage process. Since the macroscopic crack has been defined as the final stage for which $\mathbb{C}^{\text {hom }}(d)=0$ we can already anticipate that $\boldsymbol{\Sigma}^{r e s}$ and $\boldsymbol{K}$ also go to zero. Hence, as expected, we have $\boldsymbol{\Sigma}=\mathbf{0}$ in the macrocrack.

\subsection{Second macroscopic state equation}

In order to establish the second state equation an intermediate result is due. Levin's theorem allowed us to derive the macroscopic stress $\boldsymbol{\Sigma}$. The latter can also be derived from its definition (37) together with the first state equation (32) :

$$
\boldsymbol{\Sigma}=\overline{\boldsymbol{\sigma}}=(1-f) \mathbb{C}_{i s o}: \overline{\boldsymbol{\varepsilon}}^{s}+(1-f)\left(\boldsymbol{\sigma}^{0}-\boldsymbol{k} \tau\right)
$$

where the temperature and the microscopic prestress $\boldsymbol{\sigma}^{0}$ were assumed uniform. Combining (44) with the first state equation (43) and definitions (42) yields:

$$
\begin{aligned}
(1-f) \overline{\boldsymbol{\varepsilon}}^{s}=\mathbb{S}_{\text {iso }}: & \left(\mathbb{C}^{h o m}: \boldsymbol{E}+\boldsymbol{\sigma}^{0}: \mathbb{S}_{i s o}: \mathbb{C}^{h o m}\right. \\
& \left.\quad-\boldsymbol{k}: \mathbb{S}_{i s o}: \mathbb{C}^{h o m} \tau-(1-f)\left(\boldsymbol{\sigma}^{0}-\boldsymbol{k} \tau\right)\right)
\end{aligned}
$$

Let $\mathbb{X}$ be defined as :

$$
\mathbb{X}=(1-f) \mathbb{S}_{i s o}-\mathbb{S}_{i s o}: \mathbb{C}^{h o m}: \mathbb{S}_{i s o}
$$

Equation (45) reduces to:

$$
(1-f) \bar{\varepsilon}^{s}=\mathbb{S}_{\text {iso }}: \mathbb{C}^{h o m}: \boldsymbol{E}-\boldsymbol{\sigma}^{0}: \mathbb{X}+\tau \boldsymbol{k}: \mathbb{X}
$$

Combining (37) and (32) and (47) we successively obtain :

$$
S=(1-f) s^{0}+(1-f) c \tau+(1-f) \boldsymbol{k}: \overline{\boldsymbol{\varepsilon}}^{s}
$$

and

$$
S=(1-f) s^{0}-\boldsymbol{\sigma}^{0}: \mathbb{X}: \boldsymbol{k}+((1-f) c+\boldsymbol{k}: \mathbb{X}: \boldsymbol{k}) \tau+\boldsymbol{k}: \mathbb{S}_{\text {iso }}: \mathbb{C}^{h o m}: \boldsymbol{E}
$$


Recognizing the macroscopic quantity $\boldsymbol{K}$ defined in (42), the second macroscopic state equation is derived:

$$
S=S^{r e s}+C \tau+\boldsymbol{K}: \boldsymbol{E}
$$

with

$$
S^{r e s}=(1-f) s^{0}-\boldsymbol{\sigma}^{0}: \mathbb{X}: \boldsymbol{k} \quad, \quad C=(1-f) c+\boldsymbol{k}: \mathbb{X}: \boldsymbol{k}
$$

As for $\boldsymbol{\Sigma}^{\text {res }}$ and $\boldsymbol{K}$ in (43), $S^{\text {res }}$ and $C \rightarrow 0$ as $d \rightarrow 1$. It is interesting that the Maxwell symmetry between the two state equations (43) and (50) is an output of the upscaling process. It ensures the existence of a thermodynamics potential.

4.5 Macroscopic internal energy density in a damaging process

We are left with the homogenization of the internal energy density. To do so, the starting point will be :

$$
U=(1-f) \bar{u}^{s}
$$

where $u$ (resp. $U$ ) is the microscopic (resp. macroscopic) internal energy density. The above equation is the counterpart of the definition of (37). Using (32) and (33), the microscopic Helmholtz free energy density reads:

$$
\psi=\psi^{0}+\frac{1}{2} \sigma^{0}: \varepsilon+\frac{1}{2} \sigma: \varepsilon-\frac{1}{2} s \tau-\frac{1}{2} s^{0} \tau
$$

Since the internal energy density is related to $\psi$ by:

$$
u=\psi+T s=\psi+T^{0} s^{0}+\tau\left(s-s^{0}\right)+T^{0}\left(s-s^{0}\right)+\tau s^{0}
$$

Equation (53) together with (54) yields:

$$
u=u^{0}+T^{0}\left(s-s^{0}\right)+\frac{1}{2} \sigma^{0}: \varepsilon+\frac{1}{2} \sigma: \varepsilon+\frac{1}{2} \tau\left(s-s^{0}\right)
$$

According to (52), the macroscopic internal energy density is:

$$
\begin{aligned}
U=(1-f) u^{0}+T^{0}\left(S-(1-f) s^{0}\right) & +(1-f) \frac{1}{2} \sigma^{0}: \bar{\varepsilon}^{s} \\
& +(1-f) \frac{1}{2} \bar{\sigma}: \bar{\varepsilon}^{s}+\frac{1}{2} \tau\left(S-(1-f) s^{0}\right)
\end{aligned}
$$

It is readily seen, using the macroscopic constitutive law (43) and equation (47), that the macroscopic internal energy density can be expressed as :

$$
\begin{aligned}
U= & (1-f) u^{0}+\frac{1}{2} \boldsymbol{\sigma}^{0}: \mathbb{S}_{i s o}: \mathbb{C}^{h o m}: \boldsymbol{E}-\frac{1}{2} \boldsymbol{\sigma}^{0}: \mathbb{X}: \boldsymbol{\sigma}^{0}+\frac{1}{2} \boldsymbol{\Sigma}^{r e s}: \boldsymbol{E} \\
& +\frac{1}{2} \boldsymbol{E}: \mathbb{C}^{h o m}: \boldsymbol{E}+\frac{1}{2}\left(\boldsymbol{k}: \mathbb{X}: \boldsymbol{\sigma}^{0}-\boldsymbol{K}: \boldsymbol{E}+S-(1-f) s^{0}\right) \tau \\
& +T^{0}\left(S-(1-f) s^{0}\right)
\end{aligned}
$$

where we took advantage of the Hill's lemma [4]:

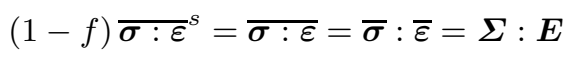


Eventually, using equation (50) together with (42) and (51), the latter can be rewritten as a function of its natural variables $\boldsymbol{E}$ and $S$ :

$$
\begin{aligned}
U= & (1-f) u^{0}-\frac{1}{2} \boldsymbol{\sigma}^{0}: \mathbb{X}: \boldsymbol{\sigma}^{0}+T^{0}\left(S-(1-f) s^{0}\right)+\frac{1}{2 C}\left(S-S^{r e s}\right)^{2} \\
+ & \boldsymbol{\Sigma}^{r e s}: \boldsymbol{E}+\frac{1}{2} \boldsymbol{E}:\left(\mathbb{C}^{\text {hom }}+\frac{1}{C} \boldsymbol{K} \otimes \boldsymbol{K}\right): \boldsymbol{E}-\frac{S-S^{r e s}}{C} \boldsymbol{K}: \boldsymbol{E}
\end{aligned}
$$

Equation (59) is similar to (24) except for the fact that the material parameters are not constant and evolve with the damage level. As stated earlier, the influence of the damage parameter is in fact hidden through the dependency of $\mathbb{C}^{\text {hom }}$ regarding $d$ which thus impacts $\boldsymbol{K}, C$ and $\mathbb{X}$.

4.6 A comprehensive micromechanics-based damage theory

In a purely micromechanics framework the state equations of the damaged REV can be derived from (43) and (50) :

$$
\left\{\begin{array}{l}
\boldsymbol{\Sigma}=\boldsymbol{\Sigma}^{\text {res }}(d)+\left(\mathbb{C}^{\text {hom }}(d)+\frac{1}{C(d)} \boldsymbol{K}(d) \otimes \boldsymbol{K}(d)\right): \boldsymbol{E}-\frac{\left(S-S^{\text {res }}(d)\right)}{C(d)} \boldsymbol{K}(d) \\
T=T^{0}+\frac{S-S^{r e s}(d)}{C(d)}-\frac{1}{C(d)} \boldsymbol{K}(d): \boldsymbol{E}
\end{array}\right.
$$

Alternatively, these equations could have been derived from a thermodynamics reasoning, where the macroscopic internal energy density $U$, derived in (59), is the relevant macroscopic potential in adiabatic conditions:

$$
\boldsymbol{\Sigma}=\frac{\partial U}{\partial \boldsymbol{E}} \quad, \quad T=\frac{\partial U}{\partial S}
$$

Recalling (23), the dissipation at the scale of the REV (therefore capital letters) is :

$$
D=\boldsymbol{\Sigma}: \dot{\boldsymbol{E}}-\dot{U}+T \dot{S} \geq 0
$$

Taking advantage of (61) when $\dot{d} \neq 0,(62)$ gives:

$$
D=-\frac{\partial U}{\partial d} \dot{d} \geq 0
$$

where $-\frac{\partial U}{\partial d}$ appears as the macroscopic thermodynamics driving force associated with the damage variable rate. A classical reasoning [9] suggests to write the damage criterion on this driving force:

$$
-\frac{\partial U}{\partial d} \leq g_{c}
$$

where $g_{c}$ is a material constant which characterizes the dissipative process and will be associated later with the creation of entropy (see subsection 6.2). 
4.7 Fully damaged macroscopic internal energy density

The sound state being a matrix without any microcracks $(f=0)$, the macroscopic elastic tensor $\mathbb{C}^{\text {hom }}$ is then simply equal to the microscopic elastic tensor $\mathbb{C}_{\text {iso. }}$. Consequently, (46) implies the nullity of $\mathbb{X}$ and the following quantities $\boldsymbol{\Sigma}^{r e s}, \boldsymbol{K}$, $S^{r e s}$ and $C$ can be replaced by $\boldsymbol{\sigma}^{0}, \boldsymbol{k}, s^{0}$ and $c$ respectively. In this case (59) takes the form (24), as expected.

The purpose of this section is to consider the opposite case of a fully damaged material. Indeed, let us recall that we have chosen to represent the 3D nucleated macrocrack as a fully damaged $(d=1)$ elastic medium. Accordingly, the expression of the internal energy $U^{F D}$, introduced in (29), is obtained by taking the limit $\mathbb{C}^{\text {hom }} \rightarrow 0$. Consequently, in a fully damaged state we have :

$$
\mathbb{C}^{h o m}=0 \quad, \quad \boldsymbol{\Sigma}^{\text {res }}=0 \quad, \quad \boldsymbol{K}=\mathbf{0} \quad, \quad \mathbb{X}=(1-f) \mathbb{S}_{\text {iso }}
$$

Besides, the expressions of $C$ and $S^{r e s}$ reduce to :

$$
S^{r e s}=(1-f) s^{0}-(1-f) \boldsymbol{\sigma}^{0}: \mathbb{S}_{i s o}: \boldsymbol{k}, C=(1-f) c+(1-f) \boldsymbol{k}: \mathbb{S}_{i s o}: \boldsymbol{k}
$$

Recalling that only the limit $f \rightarrow 0$ is relevant:

$$
\begin{aligned}
U^{F D}= & u^{0}-\frac{1}{2} \boldsymbol{\sigma}^{0}: \mathbb{S}_{\text {iso }}: \boldsymbol{\sigma}^{0}+T^{0}\left(S-s^{0}\right) \\
& +\frac{1}{2\left(c+\boldsymbol{k}: \mathbb{S}_{\text {iso }}: \boldsymbol{k}\right)}\left(S-s^{0}+\boldsymbol{\sigma}^{0}: \mathbb{S}_{\text {iso }}: \boldsymbol{k}\right)^{2}
\end{aligned}
$$

As stated earlier, we consider an adiabatic process at the macroscopic scale. Consequently, the heat transfer between the REV and its surrounding being equal to zero, the macroscopic entropy density variation between the sound and fully damage states is only the created entropy $\delta S_{c r}$. Consequently, the internal energy density in the macroscopic crack reads :

$$
U^{F D}=u^{0}-\frac{1}{2} \sigma^{0}: \mathbb{S}_{i s o}: \boldsymbol{\sigma}^{0}+T^{0} \delta S_{c r}+\frac{\left(\delta S_{c r}+\boldsymbol{\sigma}^{0}: \mathbb{S}_{i s o}: \boldsymbol{k}\right)^{2}}{2\left(c+\boldsymbol{k}: \mathbb{S}_{i s o}: \boldsymbol{k}\right)}
$$

The information regarding the dissipation in the nucleation process is characterized by $\delta S_{c r}$. In the simplified case where $\boldsymbol{\sigma}^{0}=\mathbf{0}, \delta S_{c r}$ controls the value of the residual energy (in the sense of the full damage stage). The presence of a prestress also affecting $U^{F D}$ will be discussed later (see section 5).

\subsection{Path independence}

A very instructive exercise is to verify whether or not equation (68) is compatible with the internal energy being a state function. In other words, it is interesting to see if the path independence property holds true. The proposed reasoning is sketched in Figure 4.

More precisely, let us consider an initial state (0) of the REV (related quantities with upperscript 0 ). This state is characterized by a temperature $T^{0}$, a stress state $\boldsymbol{\sigma}^{0}$, an entropy density $s^{0}$ and an internal energy density $u^{0}$. An adiabatic evolution toward a fully damaged $(F D)$ state of the REV is considered. The evolution being adiabatic, the entropy variation between states $(0)$ and $(F D)$ is a creation 
term denoted by $\delta S_{c r}$. The internal energy level expression in this fully damaged state $(F D)$ is given by (68).

Let us introduce the unloaded state $(\star)$ of the REV reached from $(0)$ by an adiabatic reversible unloading process. The temperature $T^{\star}$ reached in these adiabatic conditions is a priori different from $T^{0}$. The evolution being isentropic the entropy density in the state $(\star)$ is $s^{\star}=s^{0}$. Thus, the entropy variation between states $(\star)$ and $(F D)$ is also equal to $\delta S_{c r}$. Let $u^{\star}$ be the internal energy density in the state

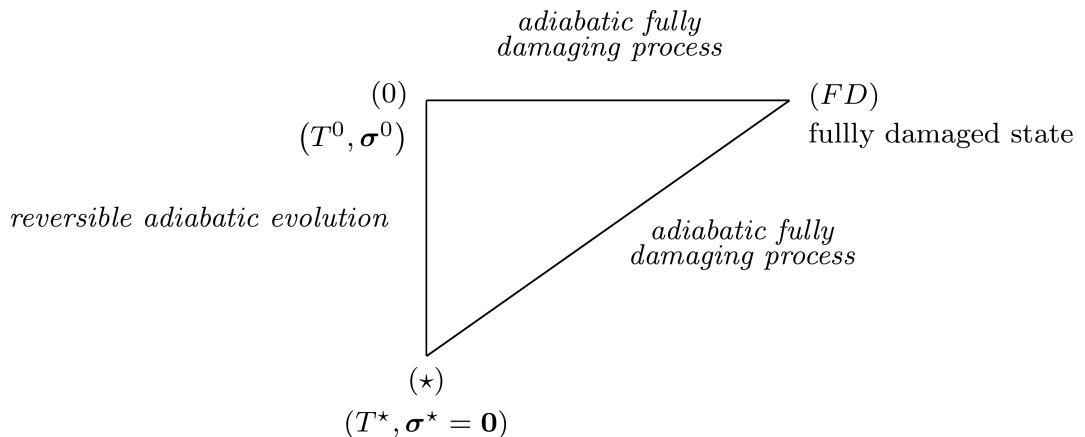

Fig. 4 Different paths leading to a fully damaged state

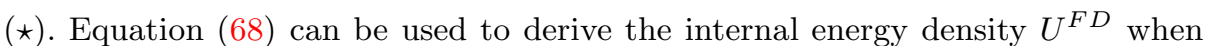
following the path $(\star) \rightarrow(F D)$, provided that $\boldsymbol{\sigma}^{0}$ and $u^{0}$ be replaced by $\boldsymbol{\sigma}^{\star}=\mathbf{0}$ and $u^{\star}$ respectively:

$$
U^{F D}=u^{\star}+T^{\star}\left(\delta S_{c r}\right)+\frac{1}{2\left(c+\boldsymbol{k}: \mathbb{S}_{i s o}: \boldsymbol{k}\right)}\left(\delta S_{c r}\right)^{2}
$$

We are now left with the assessment of the compatibility between equations (68) and (69).

The evolution $(0) \rightarrow(\star)$ being isentropic, we have :

$$
0=s^{\star}-s^{0}=c\left(T^{\star}-T^{0}\right)+\boldsymbol{k}: \varepsilon_{0}^{\star}
$$

where $\varepsilon_{0}^{\star}$ is the strain reached in the path $(0) \rightarrow(\star)$. The state $(\star)$ being unloaded the first state equation reads :

$$
0=\boldsymbol{\sigma}^{\star}=\boldsymbol{\sigma}^{0}+\mathbb{C}_{i s o}: \varepsilon_{0}^{\star}-\boldsymbol{k}\left(T^{\star}-T^{0}\right)
$$

Combining equations (70) and (71) yields :

$$
T^{\star}-T^{0}=\frac{\boldsymbol{k}: \mathbb{S}_{i s o}: \boldsymbol{\sigma}^{0}}{c+\boldsymbol{k}: \mathbb{S}_{i s o}: \boldsymbol{k}} \quad, \quad \varepsilon_{0}^{\star}=\frac{\boldsymbol{k}: \mathbb{S}_{i s o}: \boldsymbol{\sigma}^{0}}{c+\boldsymbol{k}: \mathbb{S}_{i s o}: \boldsymbol{k}} \mathbb{S}_{i s o}: \boldsymbol{k}-\mathbb{S}_{i s o}: \boldsymbol{\sigma}^{0}
$$


Between states $(0)$ and $(\star)$, the Helmholtz free energy density variation is:

$$
\begin{aligned}
\psi^{\star}-\psi^{0}=\boldsymbol{\sigma}^{0}: \varepsilon_{0}^{\star}+\frac{1}{2} \varepsilon_{0}^{\star}: \mathbb{C}_{i s o}: \varepsilon_{0}^{\star}-\left(T^{\star}\right. & \left.-T^{0}\right) \boldsymbol{k}: \boldsymbol{\varepsilon}_{0}^{\star} \\
& -\frac{c}{2}\left(T^{\star}-T^{0}\right)^{2}-s^{0}\left(T^{\star}-T^{0}\right)
\end{aligned}
$$

Recalling that $u^{\star}=\psi^{\star}+s^{\star} T^{\star}$, the associated internal energy density variation is:

$$
u^{\star}-u^{0}=\frac{1}{2} \sigma^{0}: \varepsilon_{0}^{\star}
$$

where (71) and (72) have been used. Taking advantage of (72), the latter may be reformulated as :

$$
u^{\star}=u^{0}-\frac{1}{2} \boldsymbol{\sigma}^{0}: \mathbb{S}_{i s o}: \boldsymbol{\sigma}^{0}+\frac{\left(\boldsymbol{k}: \mathbb{S}_{i s o}: \boldsymbol{\sigma}^{0}\right)^{2}}{2\left(c+\boldsymbol{k}: \mathbb{S}_{i s o}: \boldsymbol{k}\right)}
$$

Eventually, equation (69) together with (75) yield the internal energy density:

$$
\begin{aligned}
U^{F D}=u^{0}-\frac{1}{2} \boldsymbol{\sigma}^{0}: \mathbb{S}_{i s o}: & \boldsymbol{\sigma}^{0}+T^{\star} \delta S_{c r} \\
& +\frac{\left(\delta S_{c r}+\boldsymbol{k}: \mathbb{S}_{i s o}: \boldsymbol{\sigma}^{0}\right)^{2}}{2\left(c+\boldsymbol{k}: \mathbb{S}_{i s o}: \boldsymbol{k}\right)}-\frac{\boldsymbol{k}: \mathbb{S}_{i s o}: \boldsymbol{\sigma}^{0}}{c+\boldsymbol{k}: \mathbb{S}_{i s o}: \boldsymbol{k}} \delta S_{c r}
\end{aligned}
$$

At last, equation (72) allows to write the internal energy obtained when taking the path $(0) \rightarrow(\star) \rightarrow(F D)$ as :

$$
U^{F D}=u^{0}-\frac{1}{2} \sigma^{0}: \mathbb{S}_{i s o}: \boldsymbol{\sigma}^{0}+T^{0} \delta S_{c r}+\frac{\left(\delta S_{c r}+\boldsymbol{k}: \mathbb{S}_{i s o}: \boldsymbol{\sigma}^{0}\right)^{2}}{2\left(c+\boldsymbol{k}: \mathbb{S}_{i s o}: \boldsymbol{k}\right)}
$$

The former expression (68) of $U^{F D}$ is thus retrieved. This path independence is thus consistent with the internal energy being a state function.

\section{Conclusion on the macroscopic scale thermodynamics}

In order to make use of (20), it was necessary to build up a thermodynamics model of the crack. To this end, we resorted to an auxiliary density energy $U^{F D}$ related to $U_{\mathcal{F}}$ according to (29). In turn, the mathematical expression of $U^{F D}$ has been derived as a function of material constants as well as thermodynamical and mechanical quantities (68). This was the purpose of section 4.

For the sake of simplicity, $\delta S_{c r}$ is considered as a material constant. It is then readily seen that $\left(\Delta U_{\mathcal{F}}=U_{\mathcal{F}}-U_{\mathcal{F}}^{0}\right)$ :

$$
\Delta U_{\mathcal{F}}=\epsilon \int_{\mathcal{F}(\ell)}\left(-\frac{1}{2} \sigma^{0}: \mathbb{S}_{i s o}: \boldsymbol{\sigma}^{0}+T^{0} \delta S_{c r}+\frac{\left(\delta S_{c r}+\boldsymbol{\sigma}^{0}: \mathbb{S}_{i s o}: \boldsymbol{k}\right)^{2}}{2\left(c+\boldsymbol{k}: \mathbb{S}_{\text {iso }}: \boldsymbol{k}\right)}\right) d \ell
$$

Equation (20) then reduces to :

$$
\begin{aligned}
-\frac{\partial}{\partial \ell}\left(U_{\text {bulk }}-\right. & \left.U_{\text {bulk }}^{0}+K-\Phi\right)_{\mid \mathcal{C}}= \\
& \quad\left(-\frac{1}{2} \boldsymbol{\sigma}^{0}: \mathbb{S}_{\text {iso }}: \boldsymbol{\sigma}^{0}+T^{0} \delta S_{c r}+\frac{\left(\delta S_{c r}+\boldsymbol{\sigma}^{0}: \mathbb{S}_{i s o}: \boldsymbol{k}\right)^{2}}{2\left(c+\boldsymbol{k}: \mathbb{S}_{\text {iso }}: \boldsymbol{k}\right)}\right)
\end{aligned}
$$


Although $\epsilon$ is small as compared to the macroscopic structure it has a lower bound, namely the characteristic size of the heterogeneities. Therefore, it would be physically meaningless to take the limit $\epsilon \rightarrow 0$ in (79). Moreover, the right hand side of this equation is a critical energy denoted by $G_{c}^{a d}$ :

$$
-\frac{\partial}{\partial \ell}\left(U_{b u l k}-U_{b u l k}^{0}+K-\Phi\right)_{\mid \mathcal{C}}=G_{c}^{a d}
$$

Note that $G_{c}^{a d}$ might not be a material constant in case of $\boldsymbol{\sigma}^{0} \neq \mathbf{0}$.

To conclude, in adiabatic conditions, it is still possible to deal with an energy release rate provided that the isothermal elastic tensor $\mathbb{C}_{i s o}$ be replaced by the adiabatic elastic tensor $\mathbb{C}_{a d}$. Furthermore, we have to keep in mind that the right hand side of (80) is in fact derived from the internal energy contained in $\mathcal{L}_{\epsilon}$ which is a noticeable difference with the usual isothermal reasoning based on the Helmholtz free energy. From a physical point of view this difference can be explained by the fact that an isothermal crack is not able to store any Helmholtz free energy while an adiabatic crack does store internal energy. This internal energy accounts for the dissipation associated with the created entropy.

\section{Simplifications, surface density and dissipation}

\subsection{Surface density}

The purpose of this section is to provide a $2 \mathrm{D}$ interpretation of the critical energy. However, it has to be emphasized that in the proposed model we are not supposed to take the limit $\epsilon \rightarrow 0$. This can be understood as follows : if the thickness of the crack $\epsilon \rightarrow 0$, the same holds for the crack heat capacity. Since the crack has to store the dissipated energy released by nucleation, the temperature is going to rise infinite values (see Equation (90)).

Let us introduce the surface density of the created entropy $\delta S_{c r}^{2 D}$ and the surface density of the heat capacity $C^{2 D} T^{0}$. We can define those two quantities as (see Figure 5):

$$
\delta S_{c r}^{2 D}=\epsilon \delta S_{c r} \quad, \quad C^{2 D}=\epsilon C
$$

Accordingly, we derive :

$$
\begin{aligned}
& \epsilon\left(-\frac{1}{2} \boldsymbol{\sigma}^{0}: \mathbb{S}_{i s o}: \boldsymbol{\sigma}^{0}+T^{0} \delta S_{c r}+\frac{\left(\delta S_{c r}+\boldsymbol{\sigma}^{0}: \mathbb{S}_{i s o}: \boldsymbol{k}\right)^{2}}{2\left(c+\boldsymbol{k}: \mathbb{S}_{i s o}: \boldsymbol{k}\right)}\right) \\
& =-\epsilon \frac{1}{2} \boldsymbol{\sigma}^{0}: \mathbb{S}_{i s o}: \boldsymbol{\sigma}^{0}+T^{0} \delta S_{c r}^{2 D}+\frac{\left(\delta S_{c r}^{2 D}+\epsilon \boldsymbol{\sigma}^{0}: \mathbb{S}_{i s o}: \boldsymbol{k}\right)^{2}}{2 C^{2 D}}
\end{aligned}
$$

which can be simplified when the reference state does not include a prestress $\left(\sigma^{0}=0\right)$ :

$$
\epsilon\left(T^{0} \delta S_{c r}+\frac{\delta S_{c r}^{2}}{2 C}\right)=T^{0} \delta S_{c r}^{2 D}+\frac{\delta S_{c r}^{2 D^{2}}}{2 C^{2 D}}
$$

Using (82) and (83) in (79) yields :

$$
-\frac{\partial}{\partial \ell}\left(U_{b u l k}-U_{b u l k}^{0}+K-\Phi\right)_{\mid \mathcal{C}}=T_{0} \delta S_{c r}^{2 D}+\frac{\delta S_{c r}^{2 D^{2}}}{2 C^{2 D}}
$$

In conclusion, a $2 \mathrm{D}$ representation of crack consistent with the $3 \mathrm{D}$ model is provided. 


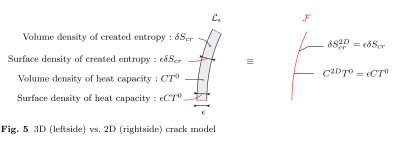

Fig. 5 3D (leftside) vs. 2D (rightside) crack model

\subsection{Dissipation}

For isothermal crack propagation, the classical reasoning is based on the notion of dissipation in order to measure the irreversibility instead of the created entropy. The quantity used to characterize the dissipation rate per unit volume associated with a damage rate $\dot{d}$ is $g_{c} \dot{d}$ and the material constant introduced is $g_{c}$ instead of $\delta S_{c r}$. However, for a fixed temperature it is readily seen that it is equivalent to deal with the notion of dissipation or the notion of created entropy. When the crack is propagating with a created length $d \ell$, the dissipation is equal to $g_{c} \times d \ell \times \epsilon \times 1$ where 1 stands for the off plan direction (it was indeed previously admitted that the crack is parametrized by $\ell$ ). Being equal by definition to $T_{0} \delta S_{c r} \times d \ell \times \epsilon \times 1$ we have :

$$
g_{c}=T_{0} \delta S_{c r}
$$

Note for forthcoming use that it is possible to define a surface density of critical energy $G_{c}=\epsilon g_{c}$. It has to be emphasized that the quantity measured experimentally is not $g_{c}$ but $G_{c}$. As such, it is rigorously equivalent in isothermal conditions to consider $\delta S_{c r}$ or $g_{c}$ as the material constant controlling the brittle behavior. This result is not surprising since the created entropy and the dissipation are related to each other through the constant temperature $T_{0}$.

If the relation between the dissipation and the created entropy is straightforward in isothermal conditions, the question may be legitimately raised to know if we could work with the dissipation in adiabatic conditions too. To do so, we need to go back to the dissipation definition $D=T \stackrel{\circ}{s r}_{c}$ and to the state equations in the damaged zone given in (60). For the sake of simplicity, we will now consider that $\boldsymbol{\sigma}^{0}=\mathbf{0}$. Since the evolution is locally adiabatic $\dot{S}=\dot{S}_{c r}$, so that the dissipation reads :

$$
D=T \dot{S}=\dot{S}\left(T^{0}+\frac{S-s^{0}-\boldsymbol{K}(d): \boldsymbol{E}}{C}\right)
$$

The dissipation at the end of the damaging process is obtained by integration of (86) from $S=s^{0}$ to $S=s^{0}+\delta S_{c r}$. It can be proved (see appendix) that $C$ can be approximated by $c$ and that the term $\boldsymbol{K}(d): \boldsymbol{E}$ may be neglected, so that the dissipation reduces to :

$$
g_{c} \dot{d}=D=\dot{S}\left(T^{0}+\frac{\left(S-s^{0}\right)}{c}\right)
$$

The integration of (87) over the whole damage process $d=0 \rightarrow 1$ yields :

$$
g_{c}=T^{0} \delta S_{c r}+\frac{\delta S_{c r}^{2}}{2 c}=U^{F D}
$$


where $U^{F D}$ is defined in (68) taking into account the assumption $\boldsymbol{\sigma}^{0}=\mathbf{0}$ and the simplification (92). Consequently, equation (84) takes the usual form :

$$
-\frac{\partial}{\partial \ell}\left(U_{b u l k}+K-\Phi\right)_{\mid \mathcal{C}}=\epsilon g_{c}
$$

Solving (88) with respect to $\delta S_{c r},(60)$ yields the expression of the temperature $T$ :

$$
T-T^{0}=\frac{\delta S_{c r}}{c}=T^{0}\left(\sqrt{1+\frac{2 g_{c}}{c T^{0^{2}}}}-1\right)=T^{0}\left(\sqrt{1+\frac{2 G_{c}}{\epsilon c T^{0^{2}}}}-1\right)
$$

This relation is in adequacy with the fact that the thinner the crack, the more important the temperature rise. From a physical point of view, this phenomenon can be explained by the fact that the internal energy variation is stored inside the crack as heat energy. As such, the heat energy will induce an increase of temperature which is controlled by the crack width.

\section{Conclusion}

This paper is motivated by the fact that nucleation is an adiabatic evolution in nature. For the same reason, it is also an undrained process if the material considered is a saturated porous solid (see [2], [7]). This also holds for dynamic propagation of existing cracks. This suggests to resort to a formulation of the energy balance in terms of internal energy instead of Helmholtz free energy. This also reveals that the crack itself possesses an internal energy which accounts for the irreversible phenomena taking place in the cracked region. The dissipation, which is very much related to isothermal processes, has to be replaced by the crack internal energy.

To understand the thermodynamics at stake, a two-scale approach is necessary. On the one hand, the crack is described by a line (resp. surface) at the macroscopic scale. This makes it possible to derive an energy balance equation of the type (80). This equation accounts at this scale for the irreversible phenomena through a linear (resp. surface) density $G_{c}^{a d}$. This quantity physically represents nothing else than the crack internal energy. On the other hand, the idea is to describe the crack at a refined scale. The crack has now a finite width and has become a structure. It is the residual state of a fully damaging process. More precisely, at this scale it is possible to refer to the constitutive material of the crack and to formulate a comprehensive micromechanics-based damage model. As an output, this upscaling process provides a micromechanics interpretation of $G_{c}^{a d}$ in terms of created entropy (see (79)). Indeed, while dissipation is most of the time introduced in isothermal evolutions, the created entropy reveals itself as the appropriate quantity to measure the irreversibility. There are experimental evidences of temperature rise during dynamic crack propagation and nucleation. The proposed theory provides the mean to evaluate this temperature rise as a function of the created entropy at the microscopic scale and the internal energy crack density at the macroscopic one. 


\section{Acknowledgment}

The authors gratefully acknowledge the reviewers for their constructive suggestions which significantly improved the paper. The authors are also grateful for the financial support by ANDRA (Agence Nationale pour la gestion des Déchets Radioactifs).

\section{Appendix}

In order to integrate (86) between the sound and the fully damaged states, it is necessary to detail the quantities $\boldsymbol{K}(d): \boldsymbol{E}$ and $C$. Hence, we will give some precisions about their order of magnitude when dealing with a claystone material. Let us consider the following orders of magnitude for such a material:

$$
c=1 \times 10^{3} \mathrm{~J} \cdot \mathrm{K}^{-2} \cdot \mathrm{m}^{-3}, \mathbb{X} \sim \mathbb{S}=1 \times 10^{-10} \mathrm{~Pa}^{-1}, \boldsymbol{k}=1 \times 10^{5} \mathrm{~Pa} \cdot \mathrm{K}^{-1}
$$

where the thermal expansion coefficient $\alpha$ is around $1 \times 10^{-5} \mathrm{~K}^{-1}$. In this framework, the following simplification may be used:

$$
k: \mathbb{X}: k \ll c
$$

Therefore $C$ can be approximated by $c$.

Damage takes place when equality in (64) is reached. Considering the internal energy derived in (59), (64) reads:

$$
-\frac{1}{2} \boldsymbol{E}: \frac{\partial \mathbb{C}_{a d}}{\partial d}: \boldsymbol{E}+\frac{S-s^{0}}{c} \frac{\partial \boldsymbol{K}}{\partial d}: \boldsymbol{E}=g_{c}
$$

Provided that the damage parameter range of variation is $O(1)$, the order of magnitude of $\frac{\partial \mathbb{C}_{a d}}{\partial d}$ and $\frac{\partial \boldsymbol{K}}{\partial d}$ is $\left|\mathbb{C}_{a d}\right|$ and $|\boldsymbol{K}|$ respectively. Taking advantage of (91) and (92), the scale analysis of (93) gives:

$$
|\boldsymbol{E}| \sim \frac{1}{\left|\mathbb{C}_{a d}\right|}\left(\frac{\left(S-s^{0}\right)|\boldsymbol{K}|}{c}\right) \Rightarrow \frac{|\boldsymbol{K}: \boldsymbol{E}|}{\left(S-s^{0}\right)} \sim \frac{|\boldsymbol{K}|^{2}}{\left|\mathbb{C}_{a d}\right| c} \ll 1
$$

\section{References}

1. B. Budiansky and R. O'Connell. Elastic moduli of a cracked solid. International Journal of Solids Structure, 12:81-97, 1976.

2. Thomas Carlioz. Nucléation et propagation de fissures en conditions anisotropes. $\mathrm{PhD}$ thesis, 2017. Paris Est.

3. L. Dormieux and D. Kondo. Micromechanics of fracture and damage. Wiley, 2016.

4. L. Dormieux, D. Kondo, and F.-J. Ulm. Microporo Mechanics. Wiley, 2006.

5. Huy Duong Bui. Fracture Mechanics: Inverse Problems and Solutions, volume 139. Springer Netherlands, 092006.

6. A. A. Griffith. The phenomenon of rupture and flow in solids. Philosophical Transactions of the Royal Society of London, 221:163-198, 1921.

7. T. Carlioz L. Dormieux, E. Lemarchand and L. Jeannin. Crack nucleation in saturated porous media. Int. Journal for Numerical and Analytical Methods in Geomechanics., 2019. in Press.

8. Dominique Leguillon. Strength or toughness? a criterion for crack onset at a notch. European Journal of Mechanics - A/Solids, 21(1):61 - 72, 2002. 
9. Jean-Jacques Marigo. Constitutive relations in plasticity, damage and fracture mechanics based on a work property. Nuclear Eng. Design, (114):249 - 272, 1989.

10. S Nemat-Nasser and M. (Muneo) Hori. Micromechanics : overall properties of heterogeneous materials. Amsterdam New York North-Holland, 1993.

11. K. Pham and J.-J. Marigo. Gradient damage models and their use to approximate brittle fracture. International Journal of Damage Mechanics, 20(4):618-652, Dec 2011.

12. Kim Pham and Jean-Jacques Marigo. Approche variationnelle de l'endommagement : I. les concepts fondamentaux. Comptes Rendus Mécanique, 338(4):191 - 198, 2010.

13. Kim Pham and Jean-Jacques Marigo. Approche variationnelle de l'endommagement : Ii. les modèles à gradient. Comptes Rendus Mécanique, 338(4):199 - 206, 2010.

14. J.R. Rice. Thermodynamics of the quasi-static growth of griffith cracks. Journal of the Mechanics and Physics of Solids, 26(2):61 - 78, 1978.

15. J. Salençon. Handbook of Continuum Mechanics. Springer, 2001.

16. Jr. Wachtman and B. John. Highlights of progress in the science of fracture of ceramics and glass. Journal of the American Ceramic Society, 57(12):509-519, 1974. 